\title{
Improvements in the Computation of Grain Opacity
}

\author{
David R. Alexander ${ }^{1}$ and Jason W. Ferguson ${ }^{2}$ \\ 1 Physics Department, Wichita State University, Wichita, Kansas 67260 USA \\ 2 Department of Physics \& Astronomy, University of Kentucky, Lexington, Kentucky \\ 40506 USA
}

\section{Introduction and Background}

Atoms and ions dominate the opacity of most astrophysical plasmas at temperatures above about $5000 \mathrm{~K}$. In the range between $5000 \mathrm{~K}$ and about $1500 \mathrm{~K}$, molecular processes are important contributors to the opacity. Depending upon the composition and pressure, grains may begin to condense at temperatures below about $1500 \mathrm{~K}$. For the conditions of interest here, the grains which form are generally small (radius $\leq 0.25 \mu \mathrm{m}$ ) compared to the wavelength of light near the peak of the Planck curve. Because their absorption and scattering cross section is so large compared to atoms or molecules, grains dominate the mean opacity whenever they are present and must, therefore, be taken into account whenever such temperatures are encountered. Our calculations indicate that failure to do so can lead to errors as large as five orders of magnitude in the mean opacity.

Spherically extended model atmospheres with $T_{\text {eff }}=2500 \mathrm{~K}$ which are either oxygen-rich (Scholz \& Tsuji 1984) or carbon-rich (Jørgensen et al. 1992) reach grain condensation conditions in their outer layers. These atmospheres are also affected by a variety of temporal phenomena, such as pulsations, shock waves, and winds, which significantly affect the condensation of grains (Fix, 1969; Sedlmayr, this volume). Since these temporal processes occur more quickly than grain condensation can reach equilibrium, the size and quantity of grains present at any given moment depends upon the past history of the atmosphere. However, the computation of the time-dependent condensation of grains is not always practical. As a first approximation, there is some utility to a general tabulation of opacities which neglects these temporal constraints and assumes that the grains are in complete thermal equilibrium with their surroundings. This assumption will form the framework for this review.

Frequency-averaged absorption coefficients are often used to reduce the radiative transfer problem to a single set of wavelength-independent equations. The Rosseland mean absorption coefficient is widely used because its use reduces the radiative transfer equation to a particularly convenient form. However, the assumption used in the derivation of the Rosseland mean that the radiation field is isotropic and Planckian breaks down if the optical depth of the medium is 
small. Under such conditions the Planck mean absorption coefficient, defined as

$$
\kappa_{\mathrm{p}}=\frac{\int \kappa_{\nu} B_{\nu} d \nu}{\int B_{\nu} d \nu}
$$

may be a more appropriate average over wavelength (see Sect. 7).

Several investigators contributed to early progress in computing grain opacity. Gaustad (1963) computed the opacity of small grains of ice, magnesium silicate, and iron, including a simple treatment of the condensation and evaporation of grains, in order to study the formation of stars. Knacke (1968) computed the absorption and scattering of $0.2 \mu \mathrm{m}$ particles of quartz and vitreous silica in response to suggestions that silicates might be constituents of the interstellar medium. Kellman \& Gaustad (1969) computed the Rosseland and Planck mean absorption coefficient for ice, vitreous silica, and graphite for use in star formation calculations.

Larger, more general tabulations of the opacity including the effects of small particles were produced by Cameron \& Pines (1973) and Alexander (1975). Both of these computations employed a simple equilibrium calculation for grains, included the opacity effects of both molecules and grains, and covered a wide range of temperatures. Alexander et al. (1983) and Pollack et al. (1985) produced even more extensive results which included detailed treatment of grain equilibrium, consideration of a wider range of grain and molecular absorbers, and the effects of the grain size distribution.

In order to compute the opacity due to small particles, one must be able to answer four sets of questions:

1. Under what conditions do the grains condense? What is their chemical composition? At a given temperature and pressure, how much of each species condenses?

2. What is the distribution of the sizes of the grains that form?

3. How do the grains absorb and scatter light?

4. What are the optical properties of the grains?

Each of these topics will be considered in turn before general results are discussed.

\section{Grain Equilibrium}

The equilibrium abundance of molecules in the gas phase is governed by the law of mass action. For example, if one considers the reaction

$$
\mathrm{A}(\mathrm{g})+\mathrm{B}(\mathrm{g}) \rightleftharpoons \mathrm{C}(\mathrm{g})
$$

then the law of mass action is a relation among the number densities of the species:

$$
\frac{n_{\mathrm{C}}}{n_{\mathrm{A}} n_{\mathrm{B}}}=K(T)
$$


where the equilibrium constant, $K(T)$, is a function of temperature only.

When grains may be present the application of the law of mass action is modified in the following way. Consider the equilibrium of corundum, relative to the monatomic gas phase,

$$
\mathrm{Al}_{2} \mathrm{O}_{3}(\mathrm{c}) \rightleftharpoons 2 \mathrm{Al}(\mathrm{g})+3 \mathrm{O}(\mathrm{g}) .
$$

The equilibrium constant for this reaction is given by

$$
K_{\text {eq }}=e^{\left(-\Delta G_{\circ} / k T\right)},
$$

where $\Delta G_{\circ}$ is the Gibbs free energy of the reaction. Corundum is condensed if

$$
K_{\mathrm{c}}=n_{\mathrm{Al}}^{2} n_{\mathrm{O}}^{3} \geq K_{\text {eq }} .
$$

In fact, just enough corundum condenses to produce an equality in Eq. (6).

If only a single substance condensed from the gas phase, the application of the above principles would be relatively simple. In reality, many species must be tested to determine if they are condensed and, if more than one species is condensed, then the abundance of all the condensates must be determined simultaneously.

Grossman (1972) presented the results of such calculations as curves showing the percentage of an element which is condensed as a function of temperature. As discussed by Alexander et al. (1983), these percentages can be subtracted from the elemental abundances and the material remaining in the gas phase can be treated with the normal equations of molecular equilibrium. Updated results (Sharp \& Huebner 1990) which include data for several chemical compositions and several pressures were used for this study.

\section{Grain Size Distribution}

Particles in an astrophysical environment will be present in a variety of different sizes. This distribution of sizes should be explicitly considered when computing the grain opacity, because the extinction efficiency of a grain depends upon its size. Mathis et al. (1977) determined that grains in the interstellar medium obeyed a power law distribution of sizes given by

$$
n(a)=k a^{-3.5},
$$

where $n(a)$ is the relative number of grains per unit volume of size $a$. Their analysis indicated that most interstellar particles occur in the size range from $0.01 \mu \mathrm{m} \leq a \leq 0.25 \mu \mathrm{m}$, except for iron grains, which they found to be six times smaller.

Seab \& Snow (1989) found that this power law distribution was consistent with observations of ultraviolet extinction around red supergiants, although they found a minimum grain size of $0.08 \mu \mathrm{m}$. Le Sergeant d'Hendecourt \& Lamy (1980) found a similar power law distribution for grain sizes, with an exponent 
of -3.85 , for small interplanetary dust particles from a study of microcraters on lunar rocks.

Dominik et al. (1989) found that the size distribution in theoretical models of the dust driven winds around cool carbon stars is represented by a power law with an exponent of -5 . While the grains in their model cover the same range of sizes as found by Mathis et al. (1977), the steeper size distribution in their model increases the total extinction due to grains by almost a factor of two.

\section{Absorption and Scattering of Light by Small Particles}

The monochromatic opacity due to absorption by grains is given by:

$$
\kappa_{\text {abs }}=\frac{\pi \sum_{a} Q_{\mathrm{abs}} n(a) a^{2}}{\rho},
$$

where $n(a)$ is the number of grains of size $a$ per cubic centimeter, $\rho$ is the total density of matter, and $Q_{\mathrm{abs}}$ is the efficiency factor for absorption. Note that if $Q_{\text {abs }}$ is equal to one, then the opacity is just equal to the geometric cross section of a grain $\left(\pi a^{2}\right)$ times the number of grains of that size, summed over the size distribution of the grains. The density appearing in Eq. (8) converts what would otherwise be a volume absorption coefficient with units of $\mathrm{cm}^{-1}$ into a mass absorption coefficient with units of $\mathrm{cm}^{2} \mathrm{~g}^{-1}$. A similar definition applies to the opacity due to scattering from grains.

Mie theory provides an exact description of the interaction of small spherical particles with electromagnetic radiation (see Bohren \& Huffman 1983). The exact treatment of scattering and absorption by small particles is only possible for a few other simple shapes. There is evidence, however, that particles in the interplanetary medium (Giese et al. 1978; Greenberg 1980), in the comae of comets (Greenberg \& Hage 1990), and in micrometeorites collected in the atmosphere (Brownlee et al. 1976) may deviate significantly from spherical shape. It is, therefore, desirable to investigate the effects of shape on grain opacity.

Hage \& Greenberg (1990) approximated non-spherical particles as aggregates of cubical subparticles in a larger cubic lattice. They found that very porous grains can produce significantly greater extinction compared to solid particles of the same total size.

Rouleau \& Martin (1991) analyzed laboratory extinction measurements of amorphous carbon particles utilizing a model of aggregated particles represented by fractal clusters of subparticles. They found significant enhancement of the extinction in the infrared for these fractal clusters compared to spherical particles. They also found that a model based upon a continuous distribution of ellipsoids (CDE), to be discussed below, is a good representation of the extinction properties determined from their much more complex calculations of fractal clusters.

Huffman (1988) also emphasized the importance of shape effects in analyzing laboratory observations of extinction for a variety of different materials. He 
showed that the CDE approximation produces a much better fit to laboratory observations than does a model based upon isolated spheres.

Because of the general applicability of the CDE approach and its computational simplicity, it has been adopted here. In order to develop the CDE approximation, we must first present the fundamental results of Rayleigh scattering theory. Approximate results for particles of arbitrary shape which are small compared to the wavelength of light can be obtained from Rayleigh scattering theory (see Van de Hulst 1957:

$$
\begin{aligned}
Q_{\mathrm{abs}} & =4 x \operatorname{Im}[\alpha] \\
\text { and } & \\
Q_{\mathrm{sca}} & =\frac{8}{3} x^{4}|\alpha|,
\end{aligned}
$$

where $x=2 \pi a / \lambda$ and $\alpha$ is the complex polarizability per unit volume of the grain material, subject to the conditions

$$
\begin{gathered}
x \ll 1 \\
\text { and } \\
x|m| \ll 1,
\end{gathered}
$$

where $m=n+i k$ is the complex index of refraction of the grain material.

For spheres the polarizability is given by

$$
\alpha=\frac{(\epsilon-1)}{(\epsilon+2)},
$$

where $\epsilon=m^{2}$ is the complex dielectric function of the grain material. For an ellipsoid (of volume $V$ ) with incident electric field parallel to a principal axis, the polarizability is

$$
\alpha=V \frac{\epsilon-1}{1+L(\epsilon-1)},
$$

where $L$ is a geometrical factor dependent solely on the shape of the ellipsoid.

The CDE model assumes that the particles are ellipsoids, with all possible shapes represented with equal probability. This distribution of shapes includes long needles, thin plates, spheres, and all other possible ellipsoidal shapes. The CDE model also assumes that these ellipsoids are oriented randomly. The result of this double averaging over both shape and orientation is that the polarizability is given in the simple form (Bohren \& Huffman 1983):

$$
\ll \alpha \gg=\frac{1}{3} \frac{2 \epsilon}{\epsilon-1} \ln \epsilon-2 .
$$

Values of the polarizability obtained from Eq. (13) can then be used directly in the Rayleigh scattering formulae given above in Eq. (9) to find the scattering 
and absorption efficiencies for the continuous distribution of randomly oriented ellipsoids. For large $x$, where Rayleigh scattering approaches infinity because Eq. (10) is not satisfied, the CDE approximation is replaced by a standard Mie calculation for spheres.

\section{Optical Properties of Materials of Interest}

The condensation equilibrium calculations described previously show that the principal high temperature $(T>600 \mathrm{~K})$ condensates will include a variety of magnesium and iron silicates, iron, carbon, and silicon carbide, depending upon the chemical composition. Specifically, silicates condense when the carbon to oxygen ratio $(C / O)$ is less than one and, under conditions of extremely high density, even when $C / O>1$. Carbon and silicon carbide only condense when $C / O>1$, while iron condenses at any value of $C / O$. At lower temperatures, other materials begin to condense and the presence of composite grains needs to be considered.

A great variety of laboratory investigations of these materials have appeared in the literature in recent years. Huffman $(1988,1989)$ emphasized the difficulties in selecting appropriate laboratory values for use in astrophysical calculations. For example, he demonstrated that optical properties derived from bulk samples may differ significantly from those derived from observations of small particles. Shape effects of the particles produced in the laboratory may also significantly affect the results, if not accounted for in the analysis. Among the factors to be considered in selecting a particular set of optical properties are:

1. Were the grains produced in the laboratory in the same range of sizes and shapes as will be found in the astrophysical context of interest?

2. Is the chemical composition and crystal structure of the grains produced in the laboratory appropriate for the astrophysical context of interest?

3 . Do the laboratory measurements include the full wavelength range of interest?

4. Does there exist a careful analysis of the laboratory data which presents results in a convenient form (e.g., index of refraction or dielectric function) for use in computing extinction efficiencies using the formulae of the previous section?

Studies of silicates include those of Day (1979), Dorschner et al. (1988), Draine \& Lee (1984), Pollack et al. (1973), and Mooney \& Knacke (1985). The optical properties deduced by Draine \& Lee (1984) for an "astrophysical silicate" have been adopted for this study because of their completeness and relevance to the astrophysical situations of interest here. Ossenkopf et al. (1992) have also synthesized the optical properties of a dirty "astrophysical silicate" utilizing both astronomical observations and laboratory studies.

For iron, laboratory investigations by Leksina \& Penkina (1967), Johnson \& Christy (1974), Gorban et al. (1975), and Tanabe et al. (1983) exist. Unfortunately, none of these studies meet all the selection criteria defined above. For 
example, both Leksina \& Penkina (1967) and Gorban et al. (1975) investigated the optical properties of bulk iron. Johnson \& Christy (1974) and Tanabe et al. (1983) fail to cover the entire wavelength range of interest. The data of Gorban et al. (1975) has been adopted for this study because of the relative completeness of their spectral coverage. The dangers of extrapolating iron data to wavelengths not included in a particular data set are discussed by Ferguson (1992). Obtaining better data for the optical properties of small iron grains should be a high priority for the astronomical community.

Carbon grains present a particularly difficult problem because they can take so many different physical structures, with sometimes significantly different optical properties. For high temperature grains, the question is whether the grains form as amorphous carbon or in the crystal structure of graphite. Rouleau \& Martin (1991) and Mathis (1988) summarize the existing evidence which supports the conclusion that carbon grains in the stellar environment will occur in the form of amorphous carbon.

Huffman (1988) emphasized the importance of particle clustering on interpreting laboratory measurements. Among the more complete studies of small particles are those of Koike et al. (1980), Bussoletti et al. (1987), Edoh (1983), and Tanabe et al. (1983). Draine \& Lee (1984) synthesized a variety of laboratory studies of graphite over a wide range of wavelengths. Rouleau \& Martin (1991) carefully analyzed the data of Bussoletti et al. (1987) to obtain optical constants for amorphous carbon in the wavelength range of interest here. The results of Rouleau \& Martin (1991) for the BE1 sample of Bussoletti et al. (1987) were used in the study reported here.

The optical properties of alpha-SiC have been studied by Phillip \& Taft (1960), Stephens (1980), Friedmann et al. (1981), and Borghesi et al. (1985). The thorough synthesis and analysis of laboratory data of Pégourié (1988) has been utilized in this study.

\section{Results}

\subsection{Shape Effects}

The effect of grain shape, as shown by differences between extinction computed for spherical grains and with the CDE approximation, affect grains of different compositions in radically different ways. Figure 1 shows these effects for the four grain species considered here. In each case the extinction cross section for the grain species under consideration is defined as

$$
C_{\mathrm{ext}}=Q_{\mathrm{ext}} \pi a^{2}
$$

where $Q_{\text {ext }}=Q_{\text {abs }}+Q_{\text {sca }}$.

Shape effects are generally unimportant for silicate grains, compared to the other grain species, although the extinction in the CDE approximation is fifty percent greater than the Mie result for wavelengths longer than $10 \mu \mathrm{m}$. The 

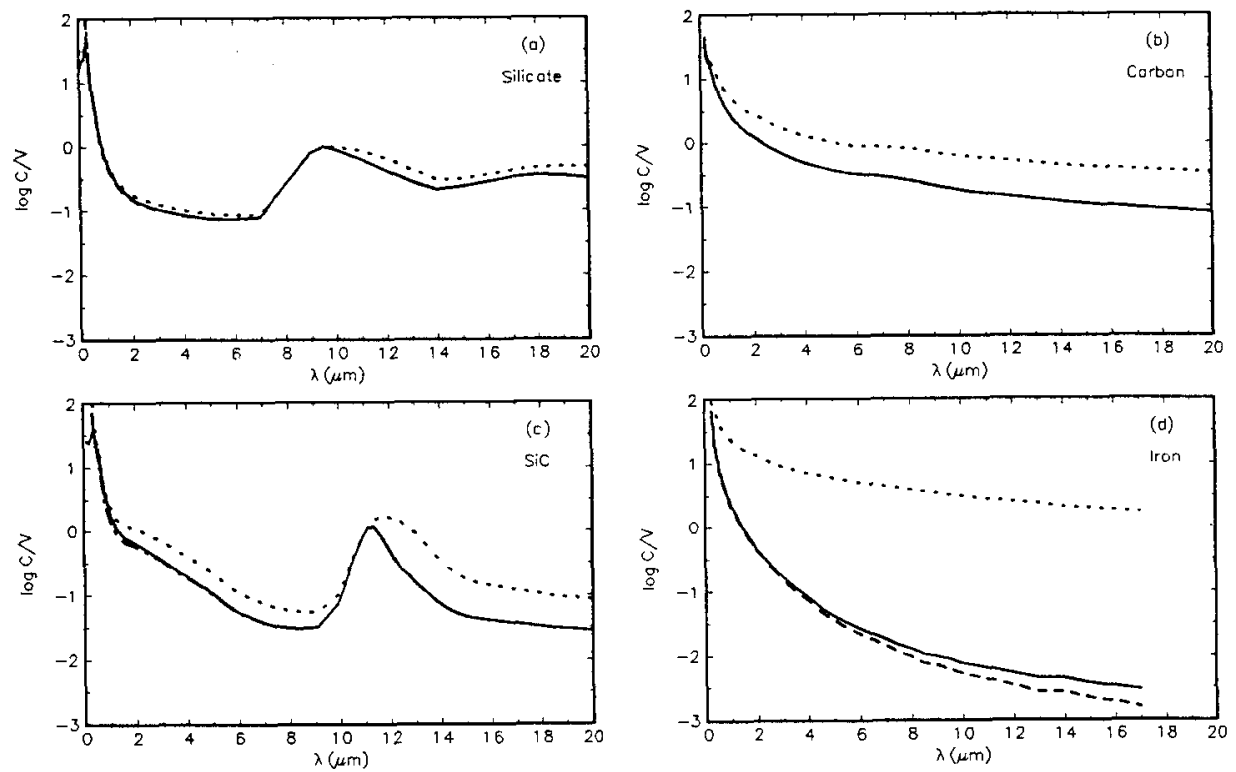

Fig. 1. Cross section for extinction per unit volume computed with Mie theory (solid curves), spherical Rayleigh particles (dashed curves), and the CDE approximation (dotted curves) for grains in the middle of the Mathis et al. (1977) size distribution.

CDE result for amorphous carbon shows a steady enhancement throughout the infrared of up to a factor of five, compared to spherical grains.

Because of the differing geometrical cross sections of grains presented to the incoming radiation field by CDE grains, sharp spectral features become more diffused in this approximation. This effect is most pronounced in the $10 \mu \mathrm{m}$ feature of silicon carbide. This effect has been noted by Bohren \& Huffman (1983) and, as they note, usually produces a better fit with laboratory data.

Shape effects are most pronounced for metallic grains. Figure 1 shows that the CDE result for iron grains is as much as three orders of magnitude greater than the Mie result. Computationally, the effect is caused by the presence of a logarithm in the computation of the polarizability in Eq. (13). When the imaginary part of the index of refraction becomes large, this term also becomes very large. It cannot be argued that the large index of refraction causes a break down in the Rayleigh approximation used in the CDE calculation, because the extinction of spheres computed with the Rayleigh approximation is actually less than the Mie result. Remember also that iron grains are smaller than the other grains considered here. 
Bohren \& Huffman (1983) find a similar effect for aluminum, which they show is necessary to explain laboratory extinction observations. Greenberg \& Hong (1975) also find that the absorption efficiency of iron needles can be up to ten times greater than the absorption efficiency of spheres.

As a cautionary note, it should be remembered that the optical properties of iron used here are derived from observations of bulk samples. If the index of refraction of small iron particles turns out to be significantly smaller than observed for bulk iron, the extinction enhancement found for the CDE result would also be greatly reduced. This reservation once again points out the importance of obtaining more appropriate optical constants for iron.

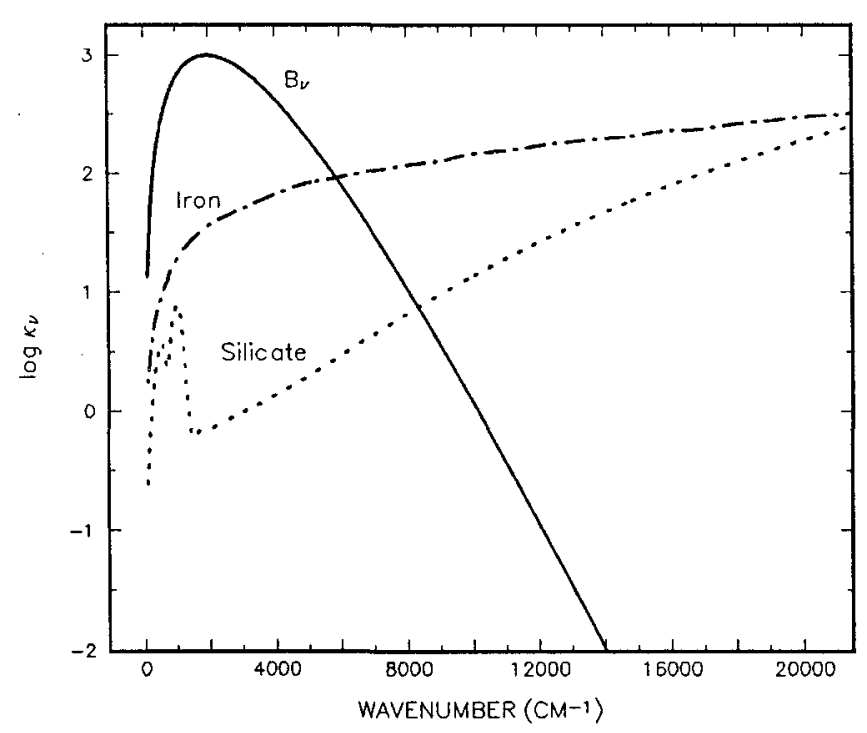

Fig. 2. Monochromatic absorption coefficients of grains for an oxygen-rich composition at $T=1000 \mathrm{~K}$ and $\rho=10^{-10} \mathrm{gm} \mathrm{cm}^{-3}$. The Planck function $B_{\nu}$, which is the weighting function for the Planck mean opacity, is shown for comparison.

\subsection{Results for $C / O<1$}

The total opacity of grains, as a function of wavenumber (defined as $1 / \lambda$ ), for the solar composition (Anders \& Grevesse, 1989) is shown in Fig. 2 for a representative value of temperature and density. Because of the CDE enhancement of the iron cross section, iron grains dominate the opacity at all wavelengths compared to silicate grains. Indeed, since iron condenses at slightly higher temperatures than silicates, iron dominates the opacity whenever grains are present at all temperatures and densities. This result is illustrated in Fig. 3, which shows the Planck mean opacity at three temperatures as a function of density. 

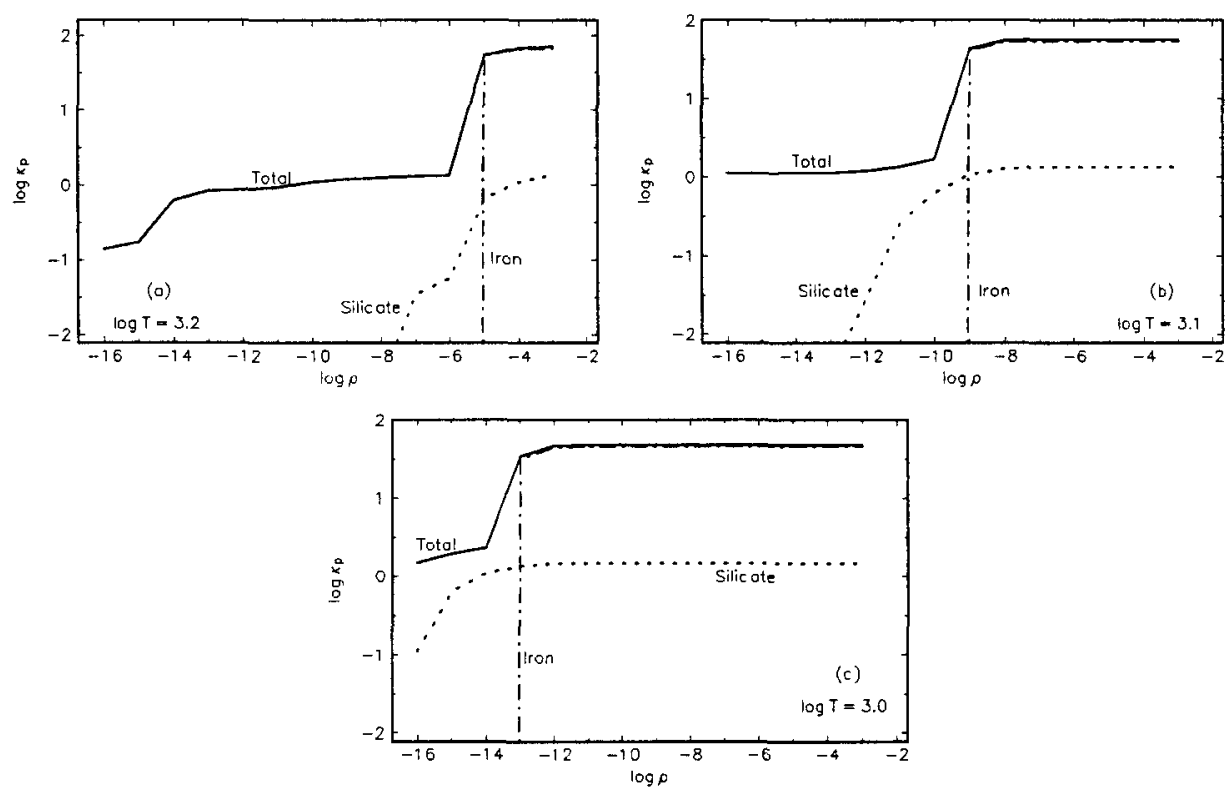

Fig. 3. The Planck mean absorption coefficient as a function of the density for three temperatures for an oxygen-rich composition. Vertical curves indicate that the species appears or disappears at that density.

\subsection{Results for $C / O>1$}

Similar results are obtained for a carbon-rich mixture $(C / O=1.05)$. Figures 4 and 5 show that iron dominates the opacity for this composition as well, as would be expected because its condensation is not strongly affected by changes in the $C / O$ ratio. The fact that silicates are present at all may seem somewhat surprising. Silicates condense under these conditions, despite the unfavourable $C / O$ ratio, because enough carbon condenses to drive the $C / O$ ratio of the gas phase below one.

Unlike the case for oxygen-rich compositions, iron does not dominate the Planck mean opacity under all conditions. Since carbon grains can form at lower densities than iron grains, the opacity at low densities is dominated by the opacity of carbon grains. 


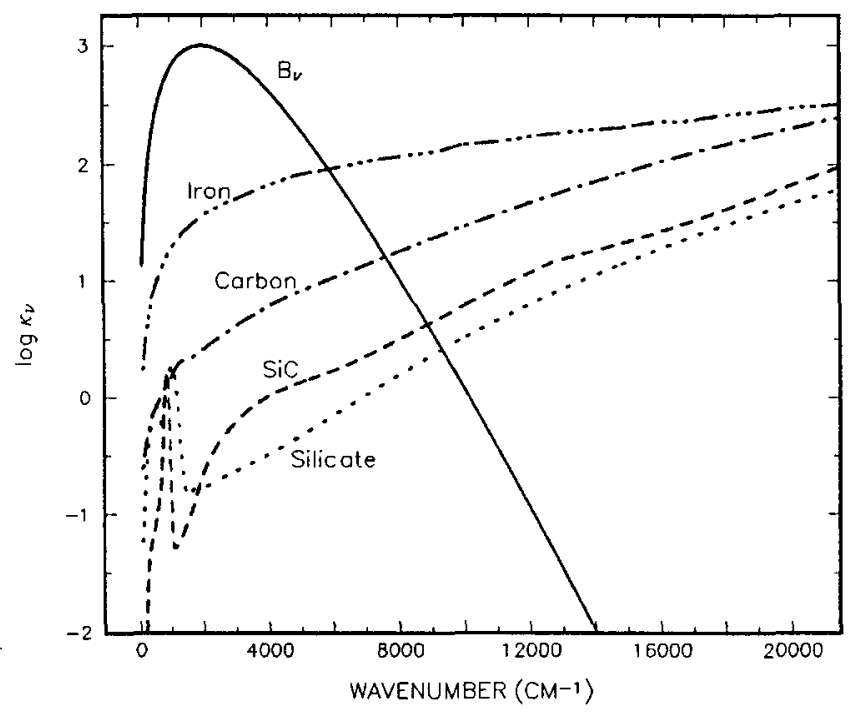

Fig. 4. Monochromatic absorption coefficient of grains for a carbon-rich composition at $T=1000 \mathrm{~K}$ and $\rho=10^{-10} \mathrm{gm} \mathrm{cm}^{-3}$. The Planck function $B_{\nu}$ is shown for comparison.

\section{Discussion and Conclusion}

The treatment of grain opacity discussed here, including the accurate treatment of condensation equilibrium, the use of a realistic distribution of grain sizes, the consideration of shape effects through the use of the CDE approximation, and the use of the most reliable optical constants available for the major condensing species, allows for the most reliable possible computation of the opacity at low temperatures. The inclusion of accurate molecular opacities, treated with the opacity sampling technique, and atomic line and continuum absorbers allows accurate opacities to be computed in the temperature range from $600 \mathrm{~K}$ to $12,000 \mathrm{~K}$ for a wide range of densities and chemical compositions. Tables of Rosseland or Planck mean opacities are available from the first author for a wide range of situations via the Internet at the address DRA @ TWSUVM.UC.TWSU.EDU.

Many factors enter into the decision of whether to use the Rosseland or the Planck mean opacity in a given situation. Clearly, model building is greatly simplified when the Rosseland mean is utilized. Nevertheless, it is important to keep in mind the nature of the average opacity one is using. Because the Rosseland mean opacity is a harmonic mean, it most strongly weights those wavelengths with small opacity. The linear Planck mean, on the other hand, more strongly weights those wavelengths with the greatest opacity. Because of the nature of the assumptions made in the derivation of the Rosseland mean, one would expect it to lead to a good approximation of the radiative transfer when the optical depth of the medium under consideration is quite large. However, if the optical depth of the medium is small, then the radiative transfer will 

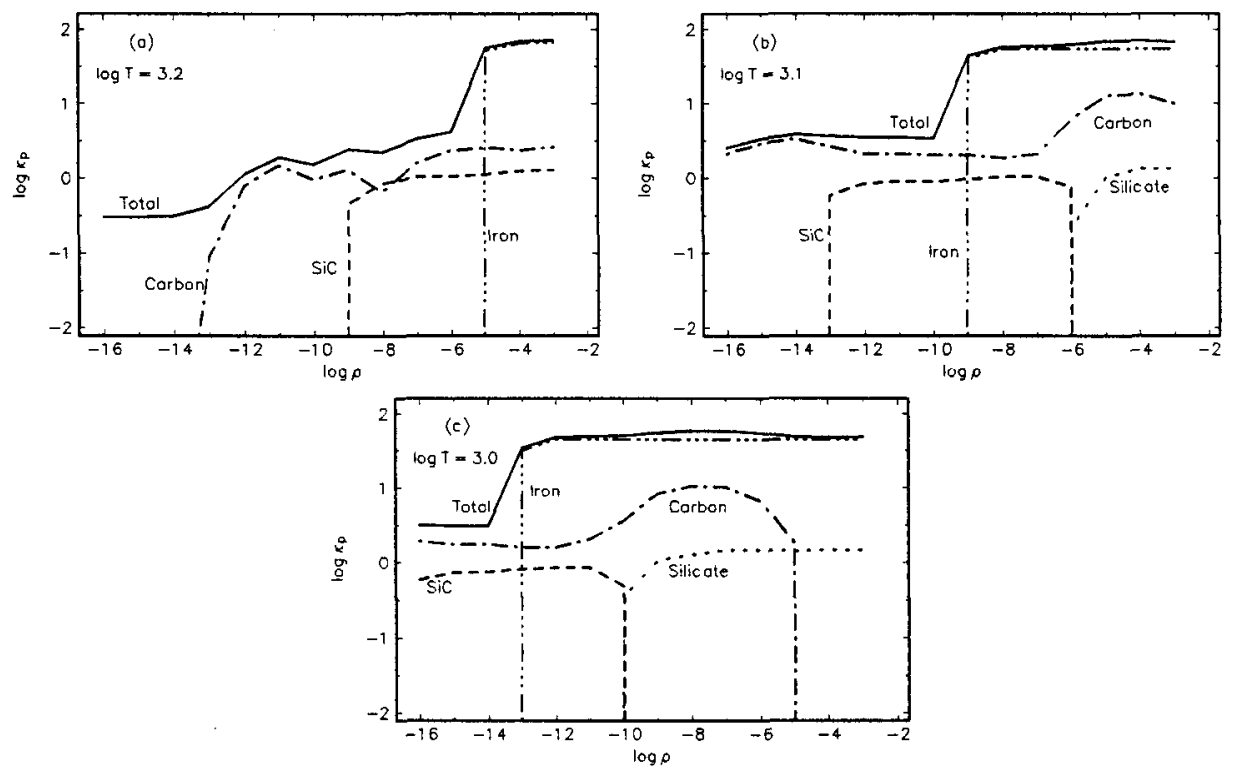

Fig. 5. The Planck mean absorption coefficient as a function of the density for three temperatures for a carbon-rich composition.

become more sensitive to the wavelengths where the opacity is highest. Hence, the Planck mean may lead to a more accurate treatment of the radiation field under those conditions than the traditional diffusion approximation (see Collins, 1989; Mihalas 1979).

Figure 6 illustrates when these considerations will be important. By comparing the ratio of the Rosseland to the Planck mean opacity, we can determine when the radiative transfer of an optically thin medium will be affected by the choice of the mean to be used. At low temperatures, where grains dominate the opacity, the two means produce virtually identical results. This result should be expected because the opacity of grains is a relatively flat and smooth function of wavelength. Similarly at high temperatures, where continuum absorption of $\mathrm{H}$ and $\mathrm{H}^{-}$dominates, the two means approach each other. However, at intermediate temperatures, where molecular line sources dominate the opacity, the two means can be orders of magnitude apart. It is in this temperature regime where these considerations will become most important. 


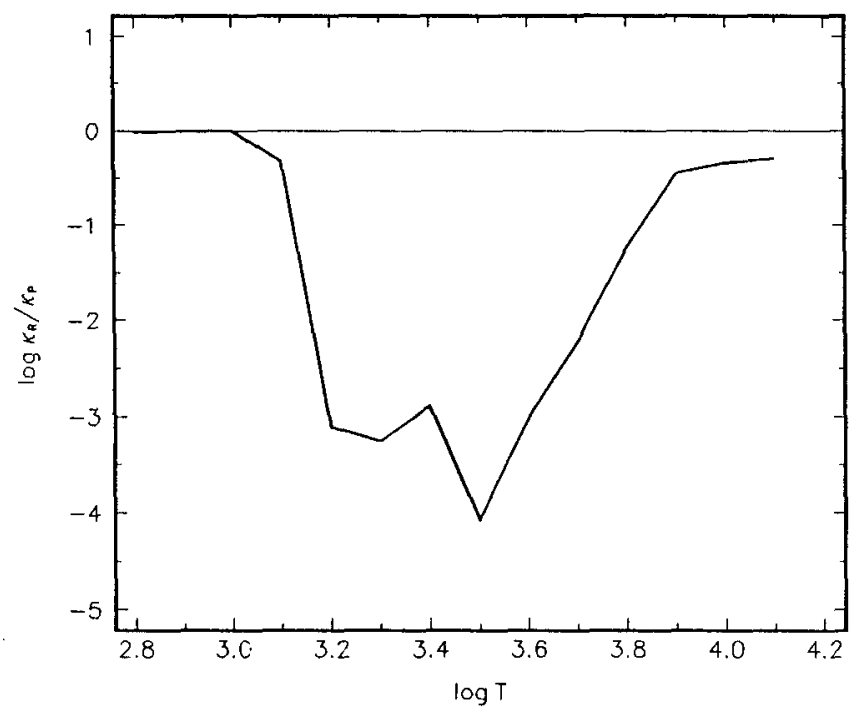

Fig. 6. The ratio of the Rosseland mean absorption coefficient to the Planck mean absorption coefficient. A reference line is shown for a ratio of one.

\section{References}

Alexander D.R., 1975, Astrophys. J. Suppl., 29, 363

Alexander D.R., Johnson H.R., Rypma R.L., 1983, Astrophys. J., 272,773

Anders E., Grevesse N., 1989, Geochim. Cosmochim. Acta, 53, 197

Bohren C.F., Huffman D.R., 1983, Absorption and Scattering of Light by Small Particles, (New York: John Wiley \& Sons), p. $82 \mathrm{ff}$

Borghesi A., Bussoletti E., Colangeli L., 1985, Astron. Astrophys., 142, 225

Bussoletti E., Colangeli L., Borghesi A., Orofino V., 1985, Astron. Astrophys. Suppl., 70, 257

Brownlee D.E., Tomandl D.A., Hodge P.W., 1976, in Interplanetary Dust and Zodiacal Light, eds. H. Elsässer and H. Fechtig, (Berlin: Springer-Verlag), 279

Cameron A.G.W., Pine M.R., 1973, Icarus, 18, 377

Collins II G.W., 1989, The Fundamentals of Stellar Atmospheres, (New York: W.H. Freeman \& Co.)

Day K.L., 1979, Astrophys. J., 234, 158

Dominik K., Gail H.-P., Sedlmayr E., 1989, Astron. Astrophys., 223, 227

Dorschner J., Friedmann C., Gürtler J., Henning T., 1988, Astron. Astrophys., 198, 223

Draine B.T., Lee H.M., 1984, Astrophys. J., 285, 89

Edoh O., 1983, Ph. D. thesis, University of Arizona, Tucson Arizona

Ferguson J. W., 1992, Masters' thesis, Wichita State University, Wichita, Kansas

Fix J.D., 1969, Mon. Not. R. Ast. Soc., 146, 37

Friedmann C., Gürtler J., Schmidt R., Dorschner J, 1981, Astrophys. Space Sci., 79, 405 
Huffman D.R., 1988, in Experiments on Cosmic Dust Analogues, eds. E. Bussoletti, C. Fusco, and G. Longo (Dordrecht: Kluwer Academic Publishers), 25

Huffman D.R., 1989, in Interstellar Dust, eds. L.J.Allamandolla and A.G.G.M. Tielens,

(Dordrecht: Kluwer Academic Publishers), 329

Gaustad J.E., 1963, Astrophys. J., 138, 1050

Giese R.H., Weiss K., Zerull R.H., Ono T., 1978, Astron. Astrophys., 65, 265

Gorban N. Y., Stashcuk V.S., Cherromorets M.F., 1975, Opt. Spectrosc., 38, 568

Greenberg J.M., 1980, in Solid Particles in the Solar System, eds. I. Halliday and B.A.

McIntosh, (Dordrecht: Reidel), 343

Greenberg J.M., Hage J.I., 1990, Astrophys. J., 361, 260

Greenberg J.M., Hong S.S., 1975, in The Dusty Universe, eds. G.B. Field and A.G.W. Cameron, (New York: Neale Watson Academic Publications, Inc.), 131

Hage J.I., Greenberg J.M., 1990, Astrophys. J., 361, 251

Grossman L., 1972, Geochim. Cosmochim. Acta, 36, 597

Johnson P.B., Christy R.W., 1974, Phys. Rev. B, 9, 5056

Jørgensen U.G., Johnson H.R., Nordlund $\AA, 1992$, Astron. Astrophys., 261, 263

Kellman S.A., Gaustad J.E., 1969, Astrophys. J., 157, 1465

Knacke R.F., 1968, Nature, 217, 44

Koike C., Hasegawa H., and Manabe A., 1980, Astrophys. Space Sci., 67, 495

Le Sergeant d'Hendecourt L.B., Lamy P.L., 1980, Icarus, 43, 350

Leksina I.Y., Penkina N.V., 1967, Phys. Metals Metallography, 23, No. 2, 344

Mathis J.S., 1988, Astro. Lett. and Comm., 26, 239

Mathis J.S., Rumpl W., Nordsieck K.H., 1977, Astrophys. J., 217, 425

Mihalas D., 1979, Stellar Atmospheres, (San Francisco: W.H. Freeman \& Co.)

Mooney T., Knacke R.F., 1985, Icarus, 64, 493

Ossen kopf V., Henning T., Mathis J.S., 1992, Astron. Astrophys., 261, 567

Pégourié B., 1988, Astron. Astrophys., 194, 335

Phillip H.R., Taft E.A., 1960 in Proc. Conference on Silicon Carbide, ed. J.S. O'Connor,

(Boston: Pergamon), 366

Pollack J.B., McKay C.P., Christofferson B.M., 1985, Icarus, 64, 471

Pollack J.B., Toon O.B., Khare B.N., 1973, Icarus, 19, 372

Rouleau F., Martin P.G., 1991, Astrophys. J., 377, 526

Seab C.G., Snow T.P., 1989, Astrophys. J., 347, 479

Scholz M., Tsuji T., 1984, Astron. Astrophys., 130, 11

Sharp C.M., Huebner W.F., 1990, Astrophys. J. Suppl., 72, 417

Stephens J.R., 1980, Astrophys. J., 237, 450

Tanabe T., Nakada Y., Kamijo F., 1983, Publ. Astron. Soc. Japan, 35, 397

Van de Hulst H.C., 1957, Light Scattering by Small Particles, (New York: John Wiley \& Sons, Inc.), p. $63 \mathrm{ff}$ 